\title{
Lebenslange Immuntherapie bei Insektengiftallergie?
}

— Wie Dr. Dorothea Wieczorek, Hannover, erläuterte wird in Deutschland mehr als die Hälfte der Bevölkerung mindestens einmal im Leben von Biene, Wespe, Hornisse oder - deutlich seltener - Hummel gestochen. Im Jahr werden hierzulande rund 3.500 Notarzteinsätze wegen eines solchen Hymenopterenstichs notwendig.

Dabei, so Wieczorek, seien Todesfälle durch das freigesetzte Toxin wenig wahrscheinlich. Schätzungen zufolge tritt der Tod durch Insektentoxin erst bei mehr als 10.000 Hornissenstichen ein, die mittlere letale Dosis LD50 liegt hier bei $10 \mathrm{mg} / \mathrm{kg}$ Körpergewicht. Beim deutlich gefährlicheren Bienengift (LD50 2,8 mg/kg Körpergewicht) sind schätzungsweise immer noch 1.330 Stiche notwendig, bevor der Tod eintritt. Viel gravierender sind hingegen anaphylaktische Reaktionen wie Juckreiz, Flush, Urtikaria, Angioödem, Nausea, Erbrechen, Defäkation, Krämpfe, Bronchospasmen und im schlimmsten Falle Atem- und Kreislaufstillstand. Die Mortalitätsrate liegt bei 0,24 pro 1.000.000 Einwohner und Jahr, was zu jährlich 10 bis 30 Todesfällen (mit vermutlich hoher Dunkelziffer) führt. Männer sind mit 68,4\% deutlich häufiger betroffen als Frauen, $95 \%$ der Todesfälle treten nach Einzelstichen auf.

Risikofaktoren für einen letalen Ausgang eines Insektenstichs sind bekannt: Alter über 40 Jahre, männliches Geschlecht, Vorerkrankungen insbesondere an Schockorganen, Bienenstich im Vergleich zu Hornissenstich, Stiche in Kopf und Hals und eine erhöhte basale Serumtryptasekonzentration, die bei circa $10 \%$ aller Insektengiftallergiker vorkommt. Somit haben laut Dr. Wieczorek schätzungsweise 2,5 Millionen Personen eine potenziell lebensbedrohliche Insektengiftallergie. Dauerhaften Schutz gebe nur eine lebenslang durchgeführte subkutane Immuntherapie (SCIT). Wird sie abgesetzt, steigt das Risiko für erneute systemische Reaktionen und schwere Reaktionen, vor allem bei Personen mit Mastzellerkrankung. Derzeit gebe es kaum Studien dazu, welche Risiken das Absetzen der SCIT mit sich bringt, schon gar keine Studien zur langfristigen Entwicklung. Die Frage, ob eine SCIT bei Insektengiftallergie lebenslang durchgeführt werden sollte oder nicht, beantwortete Wieczorek deshalb eindeutig mit "Ja".

Dem begegnete Prof. Werner Aberer, Graz, mit einem klaren "Nein". Unstrittig wirke die Immuntherapie gegen Insektengift gut, schnell und nach drei Jahren immer noch doch längst nicht bei allen Patienten. Bei den Wespenstichallergikern können demnach 91-96\% zumindest vorübergehend von ihrer Allergie befreit werden, bei den Patienten

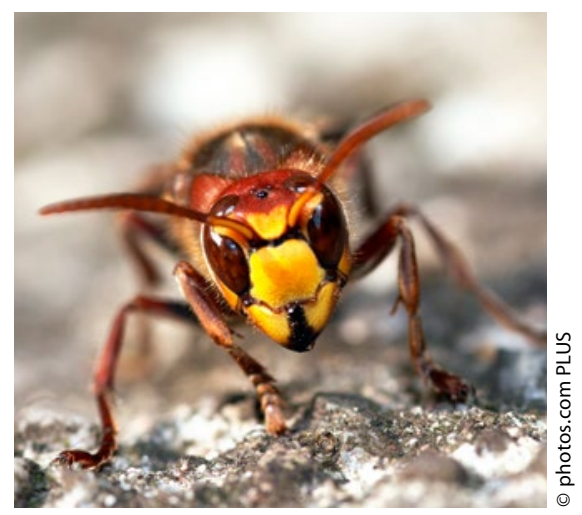

mit Bienenstichallergie sind es aber nur 77$86 \%$. Zudem gebe es dafür, ob durch die Immuntherapie noch Schutz besteht oder nicht, keinen verlässlichen Marker außer dem Stich selbst. Lebenslangen Schutz garantieren kann derzeit niemand. Diverse Untersuchungen erbrachten, dass bei milden bis mäßig starken Reaktionen drei Jahre Therapie wohl ausreichen, bei schweren Reaktionen sind vermutlich fünf Jahre notwendig. Doch keiner könne laut Aberer derzeit sagen, ob nicht auch das unzureichend sei, weshalb grundsätzlich individuelle Entscheidungen getroffen werden müssten. Aktuelle Leitlinien empfehlen die dreibis fünfährige Immuntherapie, weil kaum Evidenz für eine längere Behandlungszeit vorliegt.

Kathrin von Kieseritzky

AllergoArena: „Insektengiftallergie: Spezifische Immuntherapie lebenslang?"

\section{Allergie der Großstadt: Hygiene-These kann nicht alles erklären}

— Wie Prof. Dr. Jeroen Buters, München ausführte, steigt die Inzidenz von Asthma und Allergien auf Aeroallergene ausgehend von sehr niedrigen Werten seit den 1960erJahren stetig an, die Häufigkeit von Nahrungsmittelallergien nimmt seit den 1990erJahren zu. Dies widerlegt die These, dass in erster Linie die Einführung hygienischer Maßnahmen an der Zunahme von Allergien beteiligt ist: Die wichtigsten Veränderungen in puncto Hygiene waren in Nordeuropa und den USA bis zum Jahr 1920 vollzogen, etwa das Tragen von Schuhen als Standard, die Reinigung des Trinkwassers und bessere Wohnverhältnisse. Doch erst mehrere Jahr- zehnte später begann der Aufstieg von "Heuschnupfen", Asthma und Nahrungsmittelallergien, die heute nahezu epidemisch vorkommen. Vielmehr müssten Lebensstilveränderungen der letzten 40 Jahre als mögliche Erklärung eingehender unter die Lupe genommen werden.

Auch der simple Schluss „Bäume sind Pollenspender, also alle Bäume in der Stadt abholzen = keine Allergiker mehr" funktioniere, so Buters augenzwinkernd, nicht. Menschen lieben nunmal Bäume, und wird ein Baum in der unmittelbaren Umgebung entfernt, wirken die Bäume im weiteren Umkreis umso intensiver als Allergenspender. Studi- en zeigten zudem, dass Kinder, die schon im Babyalter vorsichtig an Allergene herangeführt werden, später seltener unter Allergien leiden. Buters Empfehlung hierzu ist einfach: „Ran an das Allergen“. Jedoch sollte in Städten eine regionale Ansammlung besonders allergenträchtiger Bäume ebenso gemieden werden wie das Anpflanzen solcher Spezies im eigenen Garten. Zudem warb Buters noch für die Installierung eines Pollenüberwachungssystems in Deutschland, dessen Ergebnisse jederzeit für die Allgemeinheit abrufbar sein sollten. Dies versetze jeden in die Lage, selbst zu entscheiden, ob und wohin der nächste Ausflug stattfinden soll. Kathrin von Kieseritzky

AllergoCompact: „Neue Aeroallergene mit regionaler, nationaler oder globaler Verbreitung" 